\title{
Conformational change in the NADP(H) binding domain of transhydrogenase defines four states
}

\author{
Vidyasankar Sundaresan, Mutsuo Yamaguchi, Justin Chartron and C. David Stout \\ Supplementary Material
}

Figure S1. Sequence Alignment of Domain III Residues in Transhydrogenase (TH)

Transhydrogenase dIII exhibits a well conserved sequence homology across various species. The sequence alignment of TH dIII ( 200 residues) from fifty-one different prokaryotic and eukaryotic species is given below, beginning at the highly conserved MNR sequence (with $\mathrm{N}$ being invariant) that is predicted to lie at the C-terminus of the last membrane-bound helix of dII (Yamaguchi, M., Stout, C.D. and Hatefi, Y. (2002) J. Biol. Chem. 277, 33670-33675; Mueller, J. and Rydström, J. (1999) J. Biol. Chem. 274, 19072-19080). The Rhodospirillum rubrum sequence (RruTHB) is given in bold type, with its numbering below the sequence alignment, arranged vertically. The sequence numbers of key residues in the protein are also given, in bold type. The sequence alignment was done using the pileup program of the GCG ${ }^{\circledR}$ Wisconsin Package ${ }^{\circledR}$ (Devereux, J., Haeberli, P., and Smithies, O. (1984) Nucleic Acids Res. 12, 387-395). 


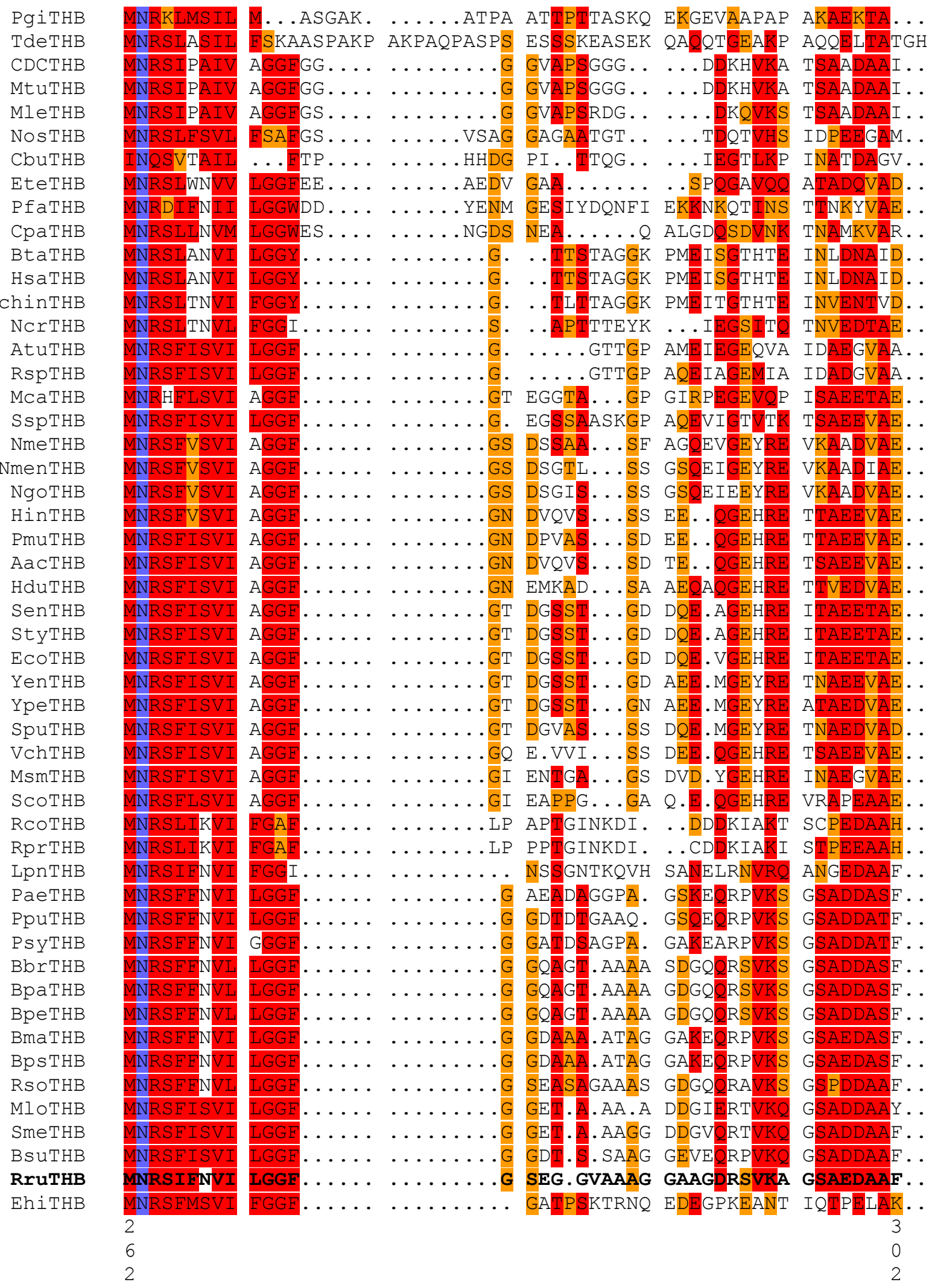




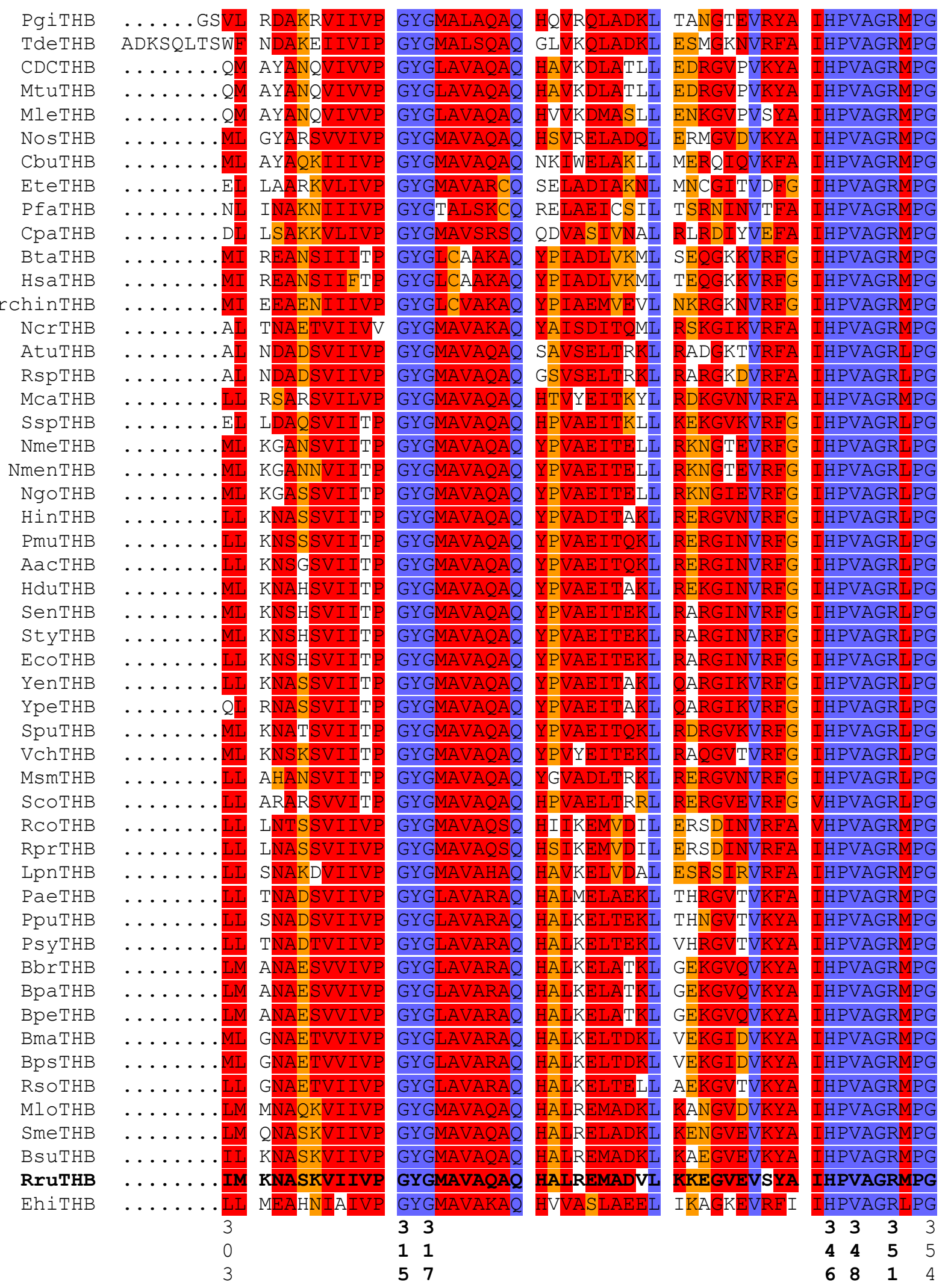




\begin{tabular}{|c|c|c|c|c|c|c|c|}
\hline $\begin{array}{l}\text { PgithB } \\
\text { TeTHB }\end{array}$ & UNNTVT T SFVD & & $H \perp$ & & $\begin{array}{l}\text { AVVVIGANDV } \\
\text { A ATT TGASDV }\end{array}$ & $\begin{array}{l}\text { NAEG } \\
\text { TAFG }\end{array}$ & $\begin{array}{l}\text { YGMPVLI } \\
\text { YGMPVTIZ }\end{array}$ \\
\hline TdeTHB & MNVLLCEVD & & & & LAII IGASDV & & $\begin{array}{l}Y G M P V L A \\
Y C M P T T A\end{array}$ \\
\hline CDCTHB & INVLLAEA] & & & & VTIVIGANDV & TNPAA & YGMPILI \\
\hline tuTHB & INVLLAEA & & $\overline{I N 1}$ & & VTIVIGANDV & TNPAA & $Y \overline{G M P I I}$ \\
\hline MletнB & INVLLAEZ & & & & VVIVIGANDI & TNPAZ & $Y \overline{G M P I}$ \\
\hline NOSTHB & MNVLLAEA & & & & VALVIGANDI & VNPA & YGMP \\
\hline CbuTHB & MNVLLAEA & & & & VAMVIGANDI & & YGLPVL \\
\hline EteTHB & MNVLLAEA & & & & VVLVVGANDT & & SGMPV \\
\hline PfaThB & LNVLLAEA & & & & IVLVVGANDI & $\mathrm{VNE}$ & ZGMPV \\
\hline Сратнв & IMNVLLAEAI & & & & VVLVIGANDT & & JGMP \\
\hline BtaThB & LNVLLAEA & & & & LVLVIGANDT & $\mathrm{VNSA}$ & \\
\hline HsathB & LNVLLAEA & & $\overrightarrow{T N}$ & & LVLVIGANDT & $\overline{V N S A}$ & \\
\hline chinthB & LNVLLAEAC & $\overline{J T, F}$ & $\overline{I N 1}$ & & LVLVIGANDT & VNSA & AGMPI \\
\hline NerTHB & DNVLLAEA & VPYI & INL & & LTLVIGANDT & $\overline{\mathrm{VNPI}}$ & AGMPVLH \\
\hline AtuTHB & HMNVLLAEA & & & & VVIVIGSNDI & VNPA & IAGMPV \\
\hline RspTHB & IMNVLLAEA & VLE] & INE & & VAIVIGSNDI & VNPAA & PIAGMPVLI \\
\hline Мсатнв & MNVLLAEA & JLEI & INE & & VAMVIGANDI & VNPAA & SPIAGMPVLI \\
\hline SspTHB & MNVLLAEA & $4 \pi \mathrm{V}$ & $\overline{I N}$ & & VVLVIGANDT & $\overline{V N P S A}$ & SAIAGMPV \\
\hline NmeTHB & MNVLLAEA & 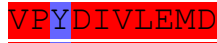 & & & VVLVIGANDT & VNPA & SPIAGMPV \\
\hline NmentHB & MNVLLAEA & $\mathrm{LE}$ & $\overline{I N}$ & & VVLVIGANDT & $\mathrm{VNPAz}$ & SPIAGMPV \\
\hline NgoTHB & MNVLLAEA & & & & VVLVIGANDT & VNPAA & SPIAGMPV \\
\hline HinTHB & MNVLLAEA & VPY & IND & & VVLVIGANDT & VNPAA & SPIAGMPVLI \\
\hline PmuTHB & MNVLLAEA & VPY & IND & & TVLVIGANDT & $\mathrm{VNPAP}$ & SPIAGMPVLI \\
\hline AacTHB & MNVLLAEA & VPY & IND & & TVLVIGANDT & VNPAAN & SPIAGMPVL \\
\hline HduTHB & $\overline{\text { MNVLLAEA }}$ & VPY & $\overline{I N 1}$ & & VVLVIGANDT & $\overline{\mathrm{VNPAA}} \mathrm{I}$ & SPIAGMPV \\
\hline SenTHB & MNVLLAEA & VPY & INI & & TVLVIGANDT & $\overline{\mathrm{VNPAA}}$ & SPIAGMPVLI \\
\hline styTHB & MNVLLAEA & VPY & INI & & TVLVIGANDT & $\overline{V N P A A Q}$ & SPIAGMPVLI \\
\hline ECOTHB & MNVLLAEA & VPY & IN1 & & $\mathrm{T}$ VLVIGANDT & VNPAAC & SPIAGMPVLI \\
\hline YenTHB & IMNVLLAEA & VPYI & IND & & VVLVIGANDT & VNPAAL & SPIAGMPVI \\
\hline YретHB & IMNVLLAEA & VPYI & IND & & VVLVIGANDT & $\overline{V N P A A}$ & SPIAGMPVLI \\
\hline SpuTHB & MNVLLAEA & VPY & $\overline{E I N E}$ & & VVLVIGANDT & VNPAAM & SPIAGMPVLI \\
\hline VchTHB & HMNVLLAEA & & $\mathrm{E} \perp \mathrm{IN}$ & & TVLVIGANDT & VNPAAL & SPIAGMPVLF \\
\hline MsmTHB & HMNVLLAEA & VPY & EINI & & VVLVIGANDT & VNPAAA & SPIAGMPVL \\
\hline SCOTHB & IMNVLLAEA & VPY & & & VVLVIGANDT & VNPSAT & SPIAGMPVLF \\
\hline RCOTHB & IMNVLLAEA & & & & VVLVIGANDV & TNPAAKNI & SPIYGMPVLI \\
\hline RprTHB & MNVLLAEA & & & & VVLVIGANDV & TNPSAKN & SPIYGMPILI \\
\hline LpnTHB & MNVLLAEA & & & & VAYVIGANDI & TNPAAKT & SPIYGMPVLF \\
\hline PaeTHB & IMNVLLAEF & & NS & & VVLVLGANDV & VNPAAKNDPK & SPIAGMPILE \\
\hline PpuTHB & IMNVLLAEA & VPYDQ & DINA & & VVLVLGANDV & VNPAAKNDPK & SPIAGMPILI \\
\hline PsyTHB & IMNVLLAEA & VPYDQ & DINS & & VVLVLGANDV & VNPAAKNDPK & SPIAGMPILI \\
\hline BbrTHB & MNVLLAEA & VPYDQ & ING & & VVLVLGANDI & VNPAAKNDPA & SPIAGMPIL \\
\hline Вратнв & MNVLLAEA & VPYDQ & $I N G$ & & VVLVLGANDI & VNPAAKNDPA & SPIAGMPIL \\
\hline Вретнв & IMNVLLAEA & VPYDQ & ING & & VVLVLGANDV & VNPAAKNDPA & SPIAGMPILI \\
\hline BmatнB & HMNVLLAEA & VPYE & ING & & VVLVLGANDV & VNPAAKNDPK & SPIAGMPIIF \\
\hline BpsthB & FMNVLLAEA & VPYE & DINGE & & VVLVLGANDV & VNPAAKNDPK & SPIAGMPII \\
\hline RsoTHB & IMNVLLAEA & VPYDQVEEN & DINSE & & VVLVLGANDV & VNPAAKNDPK & SPIAGMPILI \\
\hline MloTHB & MNVLLAEA & VPYDE VEE & DINSI & & VAYVIGANDI & TNPSARDDKS & SPIYGMPI \\
\hline SmeTHB & HMNVLLAEA & VPYDEVFEI & DINSI & & VAYVIGANDV & TNPAARDDKS & YGMP I \\
\hline BsuTHB & HMNVLLAEA & VPYDEVFELI & DINSEF & $\mathrm{AT}$ & VAFVIGANDV & TNPAAKTDPK & SPIFGMPILD \\
\hline RruTHB & HMNVLLAEAN & VPYDEVFELE & EINSSF & QTE & VAFVIGANDV & TNPAAKTDPS & SPIYGMPILD \\
\hline EhiTHB & HMNVLLAEAI & & EA.EDL & & VAIVVGANDT & & SPLAGMPI \\
\hline & 3 & & 3 & & & 3 & $\begin{array}{lll}4 & 4 & 4\end{array}$ \\
\hline & 5 & & 7 & & 9 & 9 & 00 \\
\hline & 5 & & 7 & & & & \\
\hline
\end{tabular}




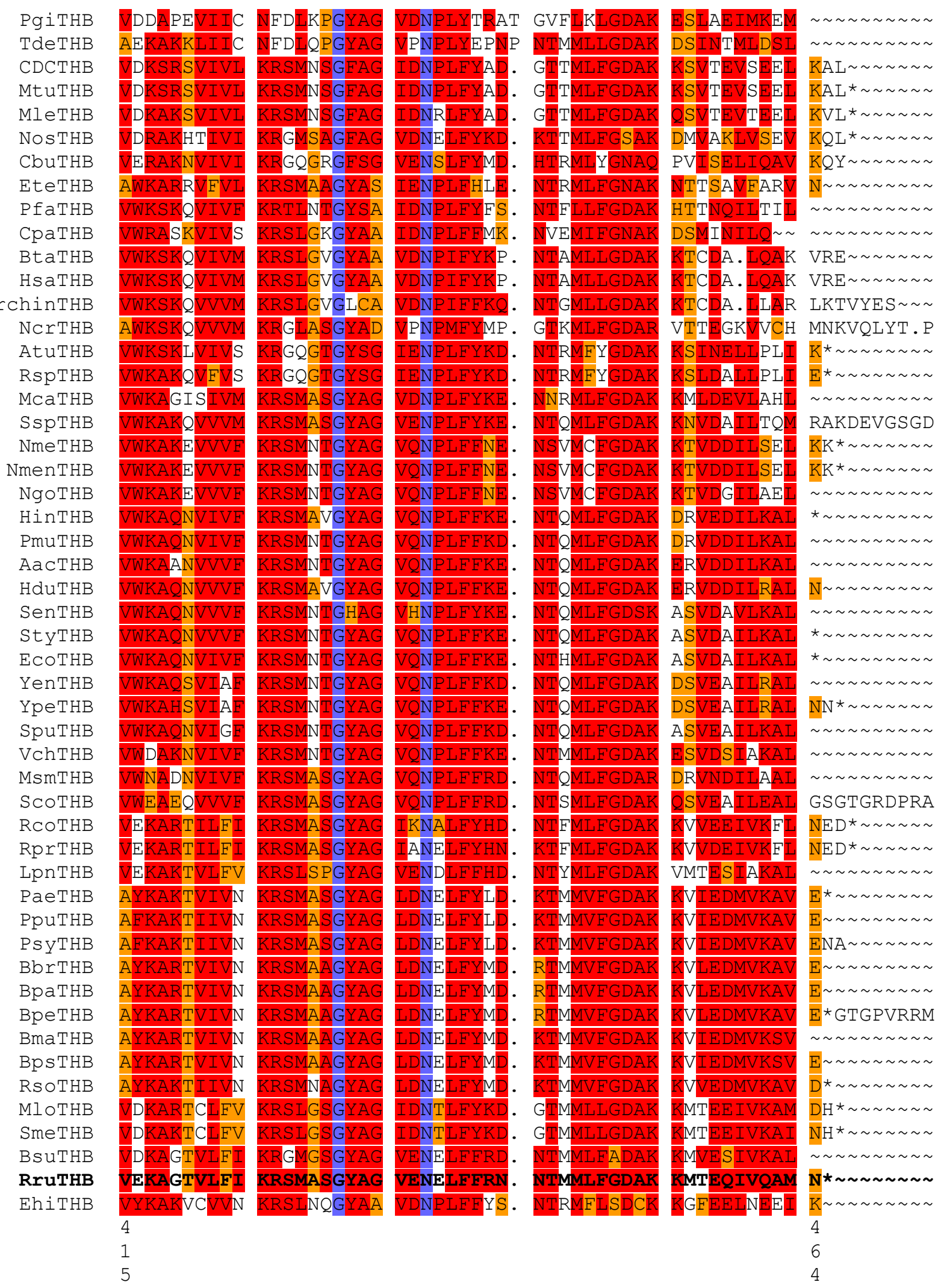




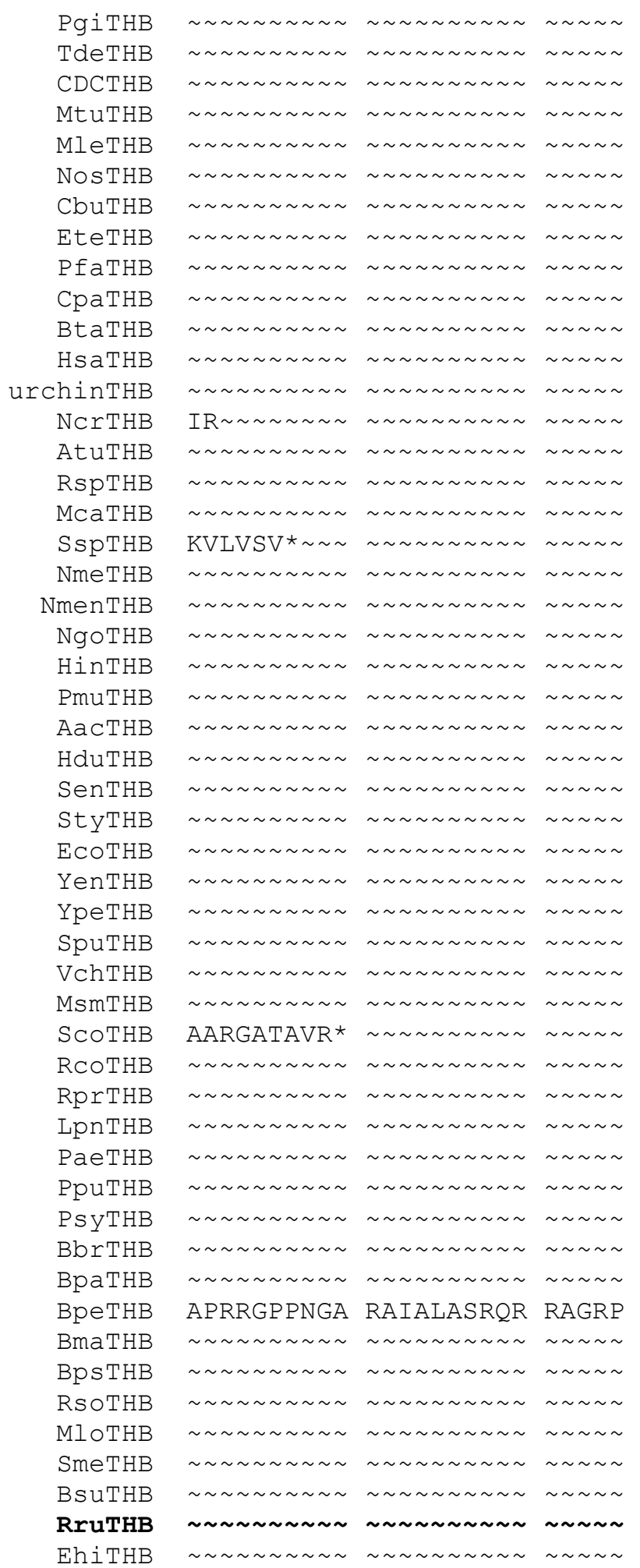

